\title{
Hair Dyeing by Using Catechinone Obtained from $(+)$-Catechin
}

\author{
YASUNAGA Hidekazu, TAKAHASHI Akiko, ITO Kazue, UEDA Masahisa, URAKAWA Hiroshi
}

Department of Biobased Materials Science, Kyoto Institute of Technology, Kyoto, Japan.

Email: yasunaga@kit.ac.jp

Received June $22^{\text {nd }}, 2012$; revised July $23^{\text {rd }}, 2012$; accepted August $10^{\text {th }}, 2012$

\begin{abstract}
A novel colourant for hair dyeing was prepared by an enzymatic reaction with $(+)$-catechin and its characteristics as a hair dyestuff were studied. The study is aimed at inventing the hair dyestuff that is human- and eco-friendly. One potentially powerful dyestuff was obtained from $(+)$-catechin treated with tyrosinase after screening experiments using biobased materials. The obtained reddish orange colourant, 4-(3,4-dihydro-3 $\alpha, 5,7$-trihydroxy- $2 H$-1-benzopyran- $2 \alpha$-yl) 1,2-benzoquinone named "catechinone", shows enough dyeability for decolourised white hair. Catechinone does not cause erythema or oedema on skin of rabbits. The colour is controlled by the addition of other biobased materials and a variety of colours of dyed hair are acquired. The colour fastness to light or washing for hair dyed by catechinone is high enough for practical use.
\end{abstract}

Keywords: Hair Dyeing; Catechinone; Dyestuff; (+)-Catechin; Biobased Materials; Safer; Human-Friendly

\section{Introduction}

The human hair dyeing by using oxidation dyes is most frequently employed throughout the world today and the number of the people dyeing their hair is increasing. The permanent hair colouring technique using the oxidation dye $[1,2]$ has advantages such as, the higher dyeability for darker hair and higher colour fastness to washing. The oxidation dyeing system generally contains dye precursors and dye couplers, which give a variety of dyestuffs while the chemical reactions proceed. The dye precursors react with oxidising agents and then work as the active intermediates that condense with the dye couplers. The addition of the dye couplers into a dyeing solution modifies the colour of dyed hair. The oxidation hair dye products also contain oxidising agents, $\mathrm{pH}$ regulators, antioxidants, preservatives, conditioning agents, surfactants and so on.

However, sensitisation symptoms are caused for some people by the use of the oxidation hair dyes [3-5]. Some of their major components and produced byproducts work as strong allergens, and the chemical action of the oxidising agent and ammonia in dyeing products is strong. One of the byproducts produced during the oxidation reaction, $p$-nitrosoaniline, causes sensitisation dermatitis with an oedema [6]. Severe dermatitis following the use of the oxidation hair dye was reported [7]. Moreover, the manufacturing process of the hair dye products and the hair dyeing are accompanied by a great amount of waste materials and waste solution.

Therefore, it is very important and desired to create a novel hair dyeing technique, which is milder and safer for a human body and is eco-friendly, in order to decrease the risks accompanying hair dyeing. Under such the situation, the authors have studied the hair dyeing by using biobased materials (natural materials). A dyestuff suitable for dyeing hair was first searched in the study. Some of substances obtained from nature are not toxic and show gentleness to a human body. Additionally, the safety of substances contained in foods and drinks for humans is relatively higher. On the other hand, the dyeability of general natural dyestuffs themselves for hair and their colour fastness are not high enough. Then, the modification of natural substances was tried to obtain an intended dye for hair colouring. Oxidised materials are generally more stable than their precursors. Therefore, colourants obtained by oxidation reaction may be stable and favourable to improving their fastness.

In the study, the oxidation of the biobased materials by the use of an enzyme was made to gain the dye, which is stable and of which dyeability is high enough. Because the toxic chemical agents for oxidation have to be removed after getting the dye, and a small amount of enzyme is deactivated in a short time and become harmless. Moreover, if the dye-production process is made concurrently with the dyeing process on the hair and skin of a scalp, the oxidation reaction should be milder. Enzymes 
work as an efficient and specific catalyst. Tyrosinase was employed in the study after the screening tests using several oxidases such as laccase and L-ascorbate oxidase. Tyrosinase acts as a monophenol monooxygenase (EC 1.14.18.1) and further oxidises catechols (EC 1.10.3.1 and EC 1.14.18.1). It is distributed in many organisms and has copper atoms at its active centre.

A number of biobased materials such as flavonoids, tannic acid, amino acids, pigments and etc. were screened to obtain the hair-dyeable colourant in the study. For example, $(+)$-catechin and other catechin derivatives are flavonoids extracted from tea plants, gambir (Uncaria gambir), areca (Areca catechu) and so on. The catechins have a catechol structure and they are oxidised by tyrosinase [8]. The the preparation, characteristics, safety, dyeability and colour fastness to washing and light for the dyestuff obtained by the enzymatic reaction of a biobased material are reported in the paper.

\section{Experimental}

\subsection{Materials}

Tyrosinase from mushroom (Sigma-Aldrich, CAS No.: 9002-10-2, $M_{\mathrm{w}}=128,000$ (obtained from the measurement of diffusion coefficient of sedimentation velocity), 133,000 (from the light scattering measurement) or 119,500 (from the electrophoresis measurement)) was used as received. $\mathrm{Na}_{2} \mathrm{HPO}_{4}$ (Nacalai Tesque, $F_{\mathrm{w}}=141.96$ ),

$\mathrm{NaH}_{2} \mathrm{PO}_{4}$ (Nacalai, $F_{\mathrm{w}}=119.98$ ) were used without further purification. $(+)$-Catechin hydrate (Sigma-Aldrich, $M_{\mathrm{w}}=290.27$ (anhydrous basis)), quercetin (Sigma-Aldrich), rutin (Nacalai Tesque), cholorogenic acid (SigmaAldrich), tannic acid (Nacalai), L-cysteine (Katayama Chemical Industries), L-tyrosine (Nacalai), L-dopa (Nacalai), naringenin (Tokyo Chemical Industry), lac dye (Kobe Chemical, KC Red EL), tamarind dye (Kobe Chem., KC Brown T), hematoxylin (Nacalai), kaoliang dye (Kiriya Chemical, Kiriyasu Brown K-12), gardenia blue (Wako Pure Chemical Industries), red cabbage dye (Kobe Chem., KC Red RA-20) were used without further purification. $p$-Aminophenol (Katayama Chem.) and 5amino-o-cresol (Tokyo Chem.) were used without further purification. Hydrogen peroxide aqueous solution (Nacalai, $30 \mathrm{w} \%$ ) was diluted to $3 \mathrm{w} \%$ with distilled water in a dye solution. The human hair samples (Matai Japan, decolourised white) were bundled by a nylon band and kept under $18 \%$ of humidity, and then were cut until just before experiments.

\subsection{Dyeing}

The enzymatic oxidation reaction of $(+)$-catechin was started by adding tyrosinase $(6640 \mathrm{U}) \mathrm{Na}_{2} \mathrm{HPO}_{4} / \mathrm{NaH}_{2} \mathrm{PO}_{4}$ buffer solution $(\mathrm{pH}=7,303 \mathrm{~K})$ into $(+)$-catechin $(1.03 \times$
$10^{-3}$ mol) $\mathrm{Na}_{2} \mathrm{HPO}_{4} / \mathrm{NaH}_{2} \mathrm{PO}_{4}$ buffer solution and the solution temperature was kept at $303 \mathrm{~K}$ throughout the dyeing. White human hair (Matai Japan, $0.7 \mathrm{~g}$ ) was immersed into the $50 \mathrm{ml}$ of mixed solution and the solution was shaken for $40 \mathrm{~min}$. The dyed hair was washed with sodium dodecyloxypolyoxyethylene sulfate solution (3 $\mathrm{wt} \%, 80 \mathrm{ml}$ ) at $308 \mathrm{~K}$ for $20 \mathrm{~min}$, rinsed twice with 500 $\mathrm{ml}$ of distilled water under at $303 \mathrm{~K}$ for $20 \mathrm{~min}$ and air-dried. The same procedure was carried out using quercetin, rutin, cholorogenic acid or tannic acid. On the other hand, the same experiments were made by the use of $(+)$-catechin and one from among L-cysteine, L-tyrosine, L-dopa, naringenin, lac dye, tamarind dye, hematoxylin, kaoliang dye, gardenia blue or red cabbage dye, in order to control the resulting colour of hair.

\subsection{Measurements}

The colour of dyed hairs were measured by a spectrocolourimeter (Konica Minolta CM-2600d) and the resulting colours were expressed in $L^{*} a^{*} b^{*}$ standard colourimetric system (CIE 1976). The $a^{*}$ and $b^{*}$ are the chromaticity coordinates. The $C^{*}$ is the chroma calculated by $C^{*}=\left\{\left(a^{*}\right)^{2}+\left(b^{*}\right)^{2}\right\}^{1 / 2}$ and $L^{*}$ is the lightness index. The colourant obtained from catechin was characterised by NMR (Bruker DRX500), MAS (JEOL JMS 700) and IR (HORIBA FT-710) measurements.

The safety test of the obtained pigment was commissioned to and made by Mitsubishi Chemical Medience Corporation as acute skin irritation study using three rabbits according to the OECD Guidelines for the testing of chemicals. The skin of rabbits was observed with the naked eye $1-72 \mathrm{~h}$ after the application of the wet pigment to the skin by using a patch.

The colour fastness to light and washing for the hair dyed by the system was examined as below. The colour fastness to light of dyed hair was estimated by irradiating the sample hairs with $9600 \mathrm{~lx}$ of daylight lamp at $301 \mathrm{~K}$ under ambient humidity. The samples were hair dyed by the dyestuff obtained from 1) (+)-catechin; 2) (+)-catechin + red cabbage; 3 ) $p$-aminophenol (PAP) +5 -aminoo-cresol (5AOC) oxidised with $\mathrm{H}_{2} \mathrm{O}_{2}$ (oxidation dye) or 4) an acid dye (commercially available hair manicure for brownish orange). The colour of irradiated hair was measured by the spectrocolourimeter at regular time intervals. The colour fastness to washing of dyed hair was also estimated by washing the sample hairs repeatedly with sodium dodecyloxypoly-oxyethylene sulfate solution (3 $\mathrm{wt} \%, 100 \mathrm{ml}$ ) at $308 \mathrm{~K}$ for $3 \mathrm{~min}$. The hair was rinsed twice with $500 \mathrm{ml}$ of distilled water at $303 \mathrm{~K}$ for $20 \mathrm{~min}$ and was air-dried. Its colour was measured by the spectrocolourimeter after every washing. 


\section{Results and Discussion}

The colour of the $(+)$-catechin solution turns momentarily orange from clear or very pale yellow by the addition of tyrosinase solution. The colour becomes deeper with time. On the other hand, the colour of the quercetin, rutin, cholorogenic acid or tannic acid solution turns pale gray or brownish-gray and deepens with time by the same procedure. The immersed hair samples are dyed orange or reddish orange in the $(+)$-catechin/tyrosinase solution, whereas they are dyed very pale yellow in the quercetin, rutin, cholorogenic acid or tannic acid with tyrosinase solution. The photographs of undyed hair and the hair treated by the $(+)$-catechin/tyrosinase system are shown in Figures 1(a) and (b), respectively. The resulting change in colour of hair, which is expressed in $L^{*} a^{*} b^{*}$ standard colourimetric system, is from $L^{*}: 78, a^{*}: 4.4, b^{*}: 21, C^{*}$ : 21 (undyed hair) to $L^{*}: 61, a^{*}: 15, b^{*}: 58, C^{*}: 60$ (dyed hair). The results show that the colourant obtained by the reaction of $(+)$-catechin with tyrosinase works as a hair dye and human hair is dyed orange.

It was revealed by thin-layer chromatography that the

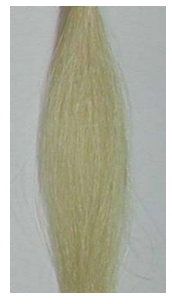

(a)

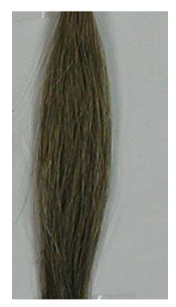

(e)

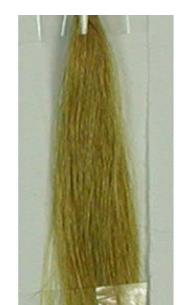

(i)

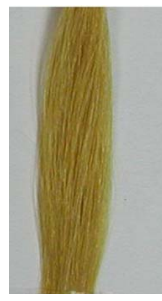

(b)

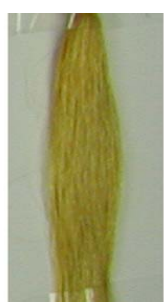

(f)

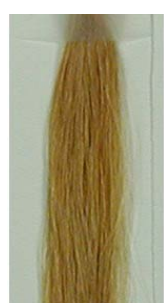

(j)

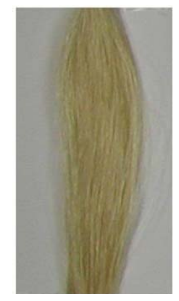

(c)

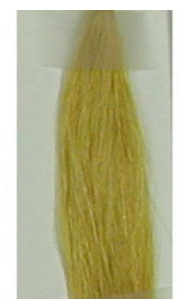

(g)

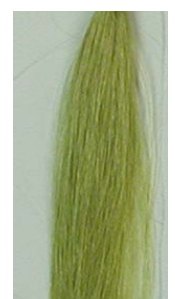

(k)

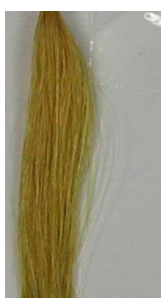

(d)

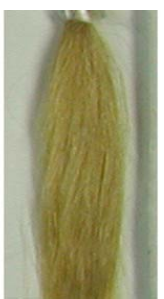

(h)

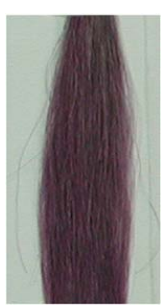

(1)
Figure 1. The photographs of undyed human hair (a) and the hair dyed by using tyrosinase with (+)-catechin (cat.) only (b), with cat. and L-cysteine (c), with cat. and L-tyrosine (d), with cat. and L-dopa (e), with cat. and naringenin (f), with cat. and lac (g), with cat. and tamarind (h), with cat. and hematoxylin (i), with cat. and kaoliang (j), with cat. and gardenia blue (k) and with cat. and red cabbage (l). obtained colourant consists of a large amount of one major component and the other minor components. The major chromatic molecule obtained is reddish powder and soluble in water and ethanol. The purification of the major pigment was attempted by recrystallisation methods. However, its perfect pure crystal was not obtained. The solubility of the pigment and $(+)$-catechin in water, ethanol and acetone is same and it is difficult to remove the trace amounts of $(+)$-catechin from the products. Then the pigment purified as highly as possible was chracterised by NMR, MAS and IR measurements. Its chemical structure determined by the characterisation is 4-(3,4-dihydro-3 $\alpha, 5,7$-trihydroxy-2H-1-benzopyran-2 $\alpha$-yl) 1,2-benzoquinone) as shown in Figure 2(b). The chemical structure of $(+)$-catechin is also shown in Figure 2 (a). The major pigment was named "catechinone" by the author. The configuration of the catechinone has not clarified. The catechinone is soluble in water and polar organic solvents such as ethanol and acetone. It was also found that white hair is dyed orange in the catechinone aqueous solution prepared from its dried powder.

The information of the influence of catechinone on a human body is important for its application to hair dyeing. The acute skin irritation test for catechinone according to the OECD Guidelines was made at the beginning. The results are shown in Table 1. It was found that no reactions on rabbits' skin such as the erythema or oedema are caused by catechinone. This shows that catechinone does not cause skin trouble under the test condition.

The control of colour of dyed hair is important and it was attempted by using biobased materials as colourcontrol additives with $(+)$-catechin. Hair was dyed with solutions prepared from $(+)$-catechin (cat.), and one of Lcysteine, L-tyrosine, L-dopa, naringenin, lac dye, tamarind dye, hematoxylin, kaoliang dye, gardenia blue or red<smiles>Oc1cc(O)c2c(c1)O[C@H](c1ccc(O)c(O)c1)[C@H](O)C2</smiles><smiles>O=C1C=CC(C2Oc3cc(O)cc(O)c3CC2O)=CC1=O</smiles>

Figure 2. Chemical structure of (+)-catechin (a) and catechinone (b). 
Table 1. Results of the acute skin irritation test for catechinone according to the OECD Guidelines. The minus (-) in the table shows no and negative reaction.

\begin{tabular}{|c|c|c|c|c|c|}
\hline \multirow{3}{*}{ Animal No. } & \multirow{3}{*}{ Reactions } & \multicolumn{4}{|c|}{ Results } \\
\hline & & \multicolumn{4}{|c|}{ Time after patch removal $/ \mathrm{h}$} \\
\hline & & 1 & 24 & 48 & 72 \\
\hline \multirow{3}{*}{1} & Erythema & - & - & - & - \\
\hline & Oedema & - & - & - & - \\
\hline & Other findings & - & - & - & - \\
\hline \multirow{3}{*}{2} & Erythema & - & - & - & - \\
\hline & Oedema & - & - & - & - \\
\hline & Other findings & - & - & - & - \\
\hline \multirow{3}{*}{3} & Erythema & - & - & - & - \\
\hline & Oedema & - & - & - & - \\
\hline & Other findings & - & - & - & - \\
\hline
\end{tabular}

cabbage dye by the treatment with tyrosinase. The dyed hairs are shown as the photographs in Figures 1(c)-(l). As a result, a variety of colours of hair are obtained as light brown for cat. + L-cysteine, yellowish brown for cat. + L-tyrosine, dark brown for cat. + L-dopa, yellow for cat. + naringenin and cat. + lac, light orange for cat. + tamarind, deep orange for cat. + hematoxylin, reddish orange for cat. + kaoliang, yellowish green for cat. + gardenia blue, and reddish violet for cat. + red cabbage. When hair is dyed only with the additive, the resulting colour is different from that of hair dyed with catechinone and the additive. The results of the measurement of the colour for the dyed hair, which are expressed in chromaticity coordinates $\left(a^{*}-b^{*}\right)$, are shown in Figure 3. The $a^{*}$ and $b^{*}$ values are widely distributed in the figure. It can be said that the colour of hair dyed by the technique is controlled by mixing (+)-catechin and bio-based materials. The colours obtained by the technique may cover the hair colours people prefer.

Moreover, the fastness of the colour of hair dyed by catechinone to light and washing is important characteristic for practical use as a hair dyeing colourant. The fastness of the colour of hair dyed by catechinone to light was first examined. The hair samples dyed by catechinone, catechinone + red cabbage, $p$-aminophenol $(\mathrm{PAP})+$ 5 -amino-o-cresol (5AOC) (oxidation dye) or an acid dye (commercially available hair manicure) were prepared. The colour of the hair dyed by catechinone and PAP + $5 \mathrm{AOC}$ were dark orange and nearly identical.

That of the hair dyed by catechinone + red cabbage was slightly purplish orange and by the acid dye was brownish orange. Figures 4 (a)-(c) show the change in

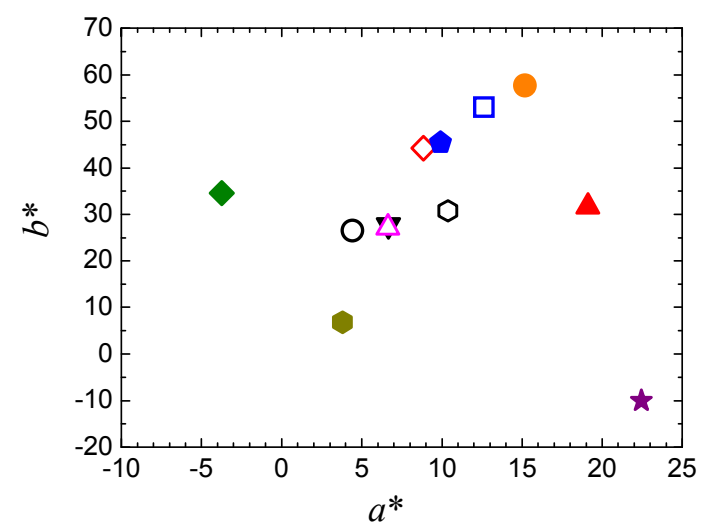

Figure 3. Chromaticity $\left(a^{*}-b^{*}\right)$ of hair dyed with (+)-catechin and biobased materials treated by tyrosinase. $\odot$ : initial, : (+)-catechin only, $\nabla$ : cat. and L-cysteine, $\square$ : cat. and Ltyrosine, $\triangle$ : cat. and L-dopa, $\diamond$ : cat. and naringenin, $\triangle$ : cat. and lac, $\bigcirc$ : cat. and tamarind, $\triangle$ : cat. and hematoxylin, $\Delta$ : cat. and kaoliang, $\diamond$ : cat. and gardenia blue, $\star$ : cat. and red cabbage.

$L^{*}, a^{*}$ and $b^{*}$ with time $(t)$ of light irradiation, respectively, for the colour of hair samples dyed by catechinone, catechinone + red cabbage, PAP +5 AOC or the acid dye. Here, the irradiation time is calculated from the integrated value of the illumination intensity and is indicated using the day unit on the basis of the assumption that hair is irradiated with mild daylight $(675 \mathrm{~lx})$ for $16 \mathrm{~h}$ per one day on average. The $L^{*}, a^{*}$ and $b^{*}$ for the hair dyed by catechinone or catechinone + red cabbage show very little change to 120 days under the light similarly to those of the hair dyed by PAP $+5 \mathrm{AOC}$ or the acid dye. These results indicate that the hair dyed by catechinone has enough fastness to daily light under the experimental condition. The colour fastness of hair dyed by catechinone to ultra violet light is under investigation by the authors.

The fastness of the colour to washing was next examined. Figures 5 (a)-(c) show the change in $L^{*}, a^{*}$ and $b^{*}$ with the number of washing $(n)$ by the use of an anionic surfactant for the colour of hair samples dyed by catechinone, catechinone + red cabbage, PAP $+5 \mathrm{AOC}$ or the acid dye. The dyed hair samples in the experiments were different from those of the light fastness experiments. The each human hair shows different dyeability and its resulted colour values $\left(L^{*}, a^{*}, b^{*}\right)$ at $n=0$ times in Figure 5 are different from the values at $t=0$ day in Figure 4. As shown in Figure 5, the $L^{*}, a^{*}$ and $b^{*}$ for the hair dyed by catechinone or catechinone + red cabbage change a little only at initial few washing. Subsequently, they change very slightly to 30 times of washing in a manner similar to those of the hair dyed by PAP + 5AOC. In contrast, the $L^{*}$ and $a^{*}$ for colour of the hair dyed by the acid dye change considerably. These results demon- 


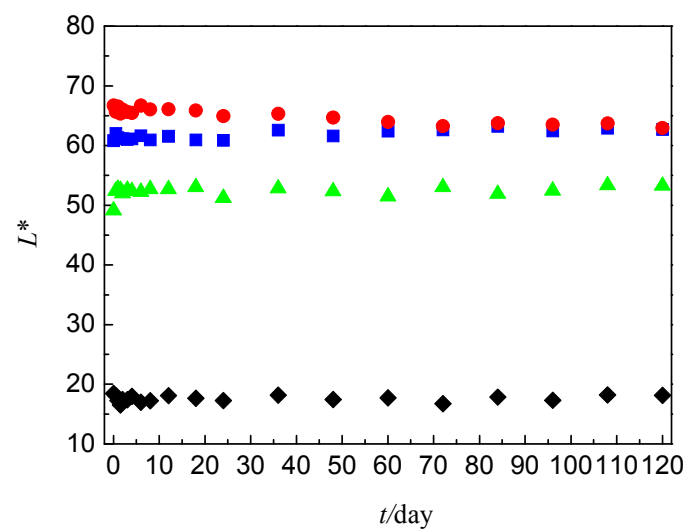

(a)

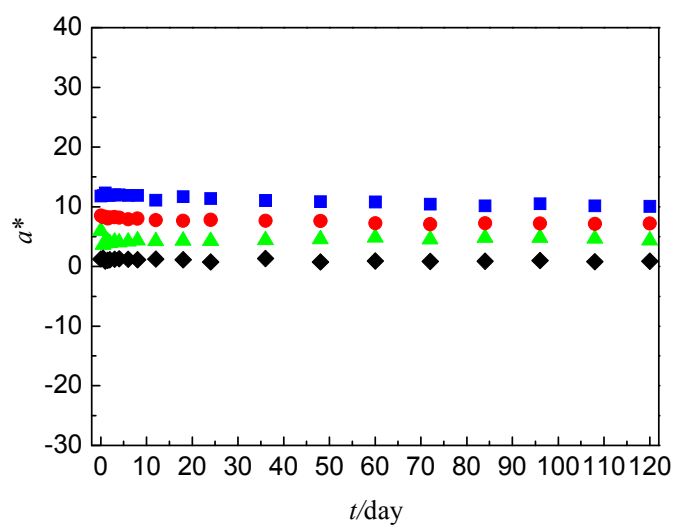

(b)

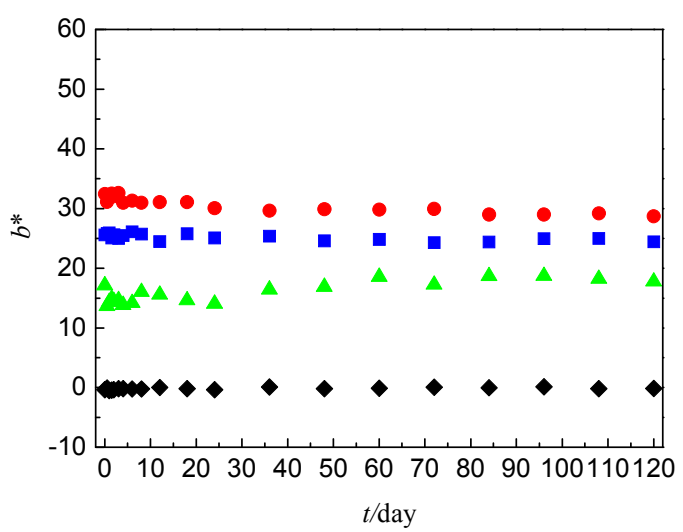

(c)

Figure 4. Change in colour $L^{*}(a), a^{*}$ (b) and $b^{*}$ (c) of hair dyed by catechinone $(\bullet)$, catechinone+red cabbage $(\triangle)$, PAP + 5AOC ( $\square)$ or acid dye $(\diamond)$ as a function of light irradiation time $(t)$. The initial value at $t=0$ day means the value for the hair just after dyed and before the irradiation.

strate that the hair dyed by catechinone has sufficient fastness to washing for practical purposes.

\section{Conclusion}

The pigment "catechinone" prepared from $(+)$-catechin

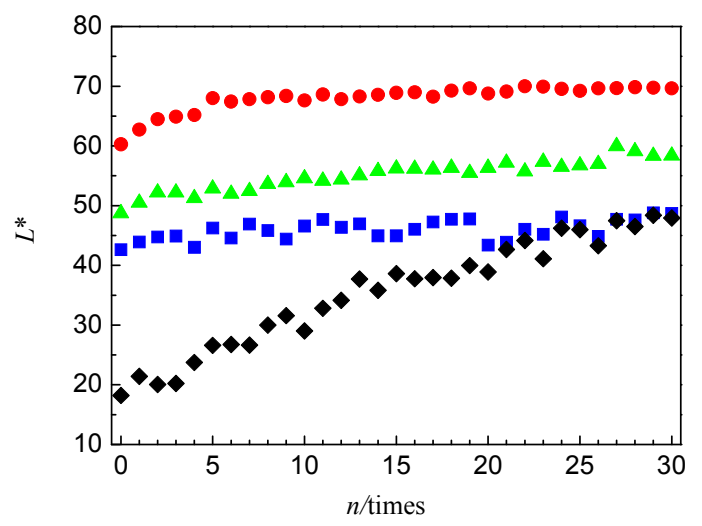

(a)

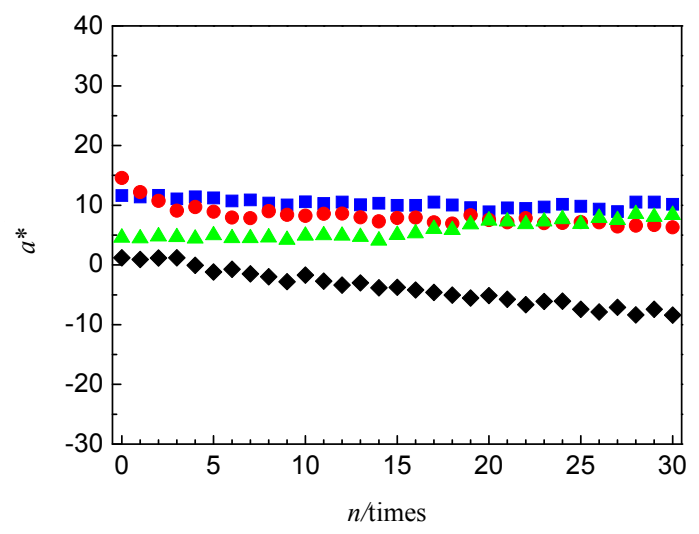

(b)

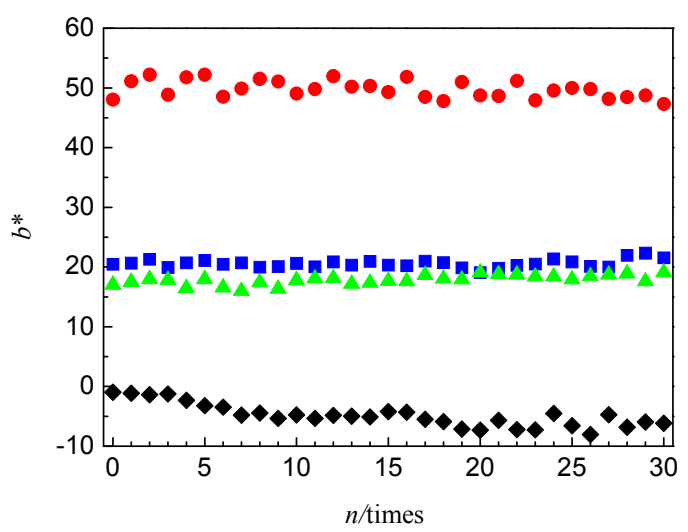

(c)

Figure 5. Change in colour $L^{*}(a), a^{*}$ (b) and $b^{*}$ (c) of hair dyed by catechinone $(\bullet)$, catechinone + red cabbage $(\triangle)$, PAP + 5AOC ( $\square)$ or acid dye $(\diamond)$ as a function of number of washing $(n)$. The initial value at $n=0$ times means the value for the hair washed once in the process of dyeing.

by the enzymatic reaction with tyrosinase, works as a hair dyestuff for decolourised white hair. Its colour is controlled by the addition of biobased materials and a variety of colours of dyed hair are acquired. Catechinone does not cause skin trouble for rabbits under the test condition. The fastness to washing or light for hair dyed by cate- 
chinone is high enough for practical use.

\section{Acknowledgements}

This study was financially supported by the Japan Society for the Promotion of Science Research Foundation Grant (No. 21500732). Japanese patent: No. 4982858.

\section{REFERENCES}

[1] J. F. Corbett, "Hair Dyes," In: K. Venkataraman, Ed., The Chemistry of Synthetic Dyes, Academic Press, Inc., New York, 1971, pp. 475-495.

[2] C. R. Robbins, "Chemical and Physical Behavior of Human Hair," 5th Edition, Springer-Verlag, Berlin, 2012, pp. 447-464. doi:10.1007/978-3-642-25611-0

[3] M. Ishihara, "Some Skin Problems Due to Hair Preparations," In: C. E. Orfanos, W. Montagna and G. Stuttgen, Eds., Hair Research: Status and Future Aspects, Proceedings of the First International Congress on Hair Research, Hamburg, 13-16 March 1981, pp. 536-542.
[4] J. Bourgeois-Spinasse, "Reactions to Hair Products," In C. E. Orfanos, W. Montagna and G. Stüttgen, Eds., Hair Research: Status and Future Aspects, Proceedings of the First International Congress on Hair Research, Hamburg, 13-16 March 1981, pp. 543-547.

[5] M. Ishihara, "Skin Hazards Due to Hair Colorings and Their Preventive Methods," Fragrance Journal, Vol. 5, No. 24, 1977, pp. 16-22.

[6] Y. Katsumura, S. Shio, T. Kobayashi and S. Fukushima, "Cross-Sensitivity of Oxidized Products of $p$-Phenylenediamine in Guinea Pigs," HiFu, Vol. 32, No. 8, 1990, pp. 54-59.

[7] H. Søsted, T. Agner, K. E. Andersen and T. Menné, “55 Cases of Allergic Reactions to Hair Dye: A Descriptive, Consumer Complaint-Based Study," Contact Dermatitis, Vol. 47, No. 5, 2002, pp. 299-303. doi:10.1034/j.1600-0536.2002.470508.x

[8] J. Oszmianski and C. Y. Lee, "Enzymatic Oxidative Reaction of Catechin and Chlorogenic Acid in a Model System," Journal of Agricultural and Food Chemistry, Vol. 38, No. 5, 1990, pp. 1202-1204. doi:10.1021/jf00095a009 